\title{
A Case of an Explicit Solutions for the Three-element Problem of Carleman Type for Analytic Functions in a Circle
}

\begin{abstract}
N. R. Perelman
Natalia R. Perelman, Smolensk State University, 4, Przheval'skogo st., 214000, Smolensk, Russia, nataly@mannet.ru

The article investigates the three-element Carleman boundary value problem in the class of analytic functions, continuous extension to the contour in the Holder sense, when this problem can not be reduced to a two-element boundary value problems. The unit circle is considered as the contour. To be specific, we study a case of inverse shift. In this case, the solution of the problem is reduced to solving a system of two integral equations of Fredholm second kind; thus significantly used the theory of F. D. Gakhov about Riemann boundary value problem for analytic functions. Based on this result, an algorithm for the solution of the problem is built. Then it is proved that if the boundary condition coefficients are rational functions, and shift a linear-fractional function, then the boundary value problem is solved in an explicit form (in quadrature). Then we consider a simple case of an explicit solution of the problem, when in addition to the above restrictions on the coefficients and shift function is required also analytic continuation of some functions defined on the contour, inside the area. This case is illustrated by a concrete example.
\end{abstract}

Key words: boundary value problem, Carleman shift.

\section{References}

1. Perelman N. R., Rasulov K. M. Three-element problem of Carleman type for bianalitic functions in a circle. Izv. Saratov Univ. (N. S.), Ser. Math. Mech. Inform., 2012, vol. 12, iss. 2, pp. 18-26 (in Russian).

2. Litvinchuk G. S. Solvability theory of boundary value problems and singular integral equations with shift. Dordrecht; Boston, Kluwer Academic Publ., 2000, 378 p. DOI: 10.1007/978-94-0114363-9. (Russ. ed. : Litvinchuk G. S. Kraevye zadachi i singuliarnye integral'nye uravneniia so sdvigom. Moscow, Nauka, 1977, 448 p.)
3. Perelman N. R., Rasulov K. M. Three-element Carleman boundary value problem with a reverce shift for analytic functions on an exceptional case. Izv. Brjanskogo Gos. Univ. [The Bryansk State Univ. Herald], 2012, no. 4(2), pp. 46-53 (in Russian).

4. Rasulov K. M. Metod soprjazhenija analiticheskih funkcij $i$ nekotorye ego prilozhenija[Conjugation method of analytic functions and some of its applications]. Smolensk, SmolGU Publ., 2013, 189 p. (in Russian).

УДК 517.927.25

\section{РАЗЛОЖЕНИЕ ПО КОРНЕВЫМ ФУНКЦИЯМ СИЛЬНО НЕРЕГУЛЯРНОГО ПУЧКА ДИФФЕРЕНЦИАЛЬНЫХ ОПЕРАТОРОВ ВТОРОГО ПОРЯДКА С КРАТНЫМИ ХАРАКТЕРИСТИКАМИ}

\begin{abstract}
В. С. Рыхлов
Рыхлов Виктор Сергеевич, кандидат фризико-математических наук, доцент кафредры дифрференциальных уравнений и прикладной математики, Саратовский национальный исследовательский государственный университет имени Н. Г. Чернышевского, RykhlovVS@yandex.ru

Рассматривается квадратичный сильно нерегулярный пучок обыкновенных диффференциальных операторов второго порядка с постоянными коэсрфициентами и с кратным корнем характеристического уравнения. Находятся суммы двукратных разложений в биортогональный ряд Фурье по корневым функциям таких пучков и, как следствие, необходимое и достаточное условие сходимости указанных разложений к разлагаемой вектор-фрункции. Это необходимое и достаточное условие является диффреренциальным уравнением, связывающим компоненты разлагаемой вектор-фрункци. При этом на разлагаемую вектор-фрункцию накладываются некоторые условия гладкости и требования обращения в нуль ее компонент и некторых их производных на концах основного отрезка.
\end{abstract}

Ключевые слова: квадратичный пучок дифрференциальных операторов, кратная характеристика, кратный корень характеристического уравнения, сильно нерегулярный пучок, двукратное разложение по собственным функциям, двукратное разложение по корневым элементам, биортогональный ряд по корневым элементам, производные цепочки, условия кратной разложимости.

DOI: 10.18500/1816-9791-2016-16-2-165-174 


\section{1. ПОСТАНОВКА ЗАДАЧИ И КРАТКАЯ ИСТОРИЯ ВОПРОСА}

Рассмотрим в пространстве $L_{2}[0,1]$ квадратичный пучок $L(\lambda)$ дифференциальных операторов 2-го порядка

$$
\begin{gathered}
\ell(y, \lambda):=y^{\prime \prime}-2 \lambda y^{\prime}+\lambda^{2} y, \\
U_{1}(y):=y(0)=0, \quad U_{2}(y):=y(1)-y^{\prime}(0)=0 .
\end{gathered}
$$

Характеристическое уравнение $\omega^{2}-2 \omega+1=0$ пучка имеет кратные корни $\omega_{1}=\omega_{2}=1$. Обозначим $y_{1}(x, \lambda)=e^{\lambda x}, y_{2}(x, \lambda)=x e^{\lambda x}$. Функции $y_{1}, y_{2}$ образуют фундаментальную систему решений уравнения $\ell(x, \lambda)=0$.

Характеристический определитель пучка $L(\lambda)$

$$
\Delta(\lambda):=\left|\begin{array}{ll}
U_{1}\left(y_{1}\right) & U_{1}\left(y_{2}\right) \\
U_{2}\left(y_{1}\right) & U_{2}\left(y_{2}\right)
\end{array}\right| \equiv e^{\lambda}-1 \equiv \Delta_{0}^{-}(\lambda) \equiv e^{\lambda}\left(1-e^{-\lambda}\right) \equiv e^{\lambda} \Delta_{0}^{+}(\lambda)
$$

является вырожденным (отсутствует слагаемое с $e^{2 \lambda}$ ), а пучок $L(\lambda)$, таким образом, сильно нерегулярным (с.н.) (определение регулярных, почти регулярных, нормальных или слабо нерегулярных пучков см. в $[1,2]$; все остальные пучки называются с.н.). Собственные значения (с.з.) $\lambda_{k}$ пучка (нули (3)) простые и $\lambda_{k}=2 k \pi i, k \in \mathbb{Z}$.

Решается задача нахождения условий на вектор-функцию (в.-ф.) $f=\left(f_{1}, f_{2}\right)^{T}$, при которых имеет место двукратная разложимость этой в.-ф. в биортогональный ряд Фурье по корневым элементам (к.э.) пучка $L(\lambda)$.

Задачи о разложении для простейших с.н. дифференциальных операторов 1-го и 2-го порядков со знакопеременной весовой функцией были решены в [3]. А именно рассматривались операторы

$$
y^{\prime}-\lambda p(x) y, \quad y(0)=y(1)
$$

и

$$
y^{\prime \prime}-\lambda p(x) y, \quad y(0)+a y(1)=0, \quad y^{\prime}(0)+i a y^{\prime}(1)=0 \quad(a \neq 0),
$$

где $p(x)=1$ при $x \in[0, \alpha]$ и $p(x)=-1$ при $x \in[\alpha, 1](0<\alpha<1 / 2$ или $1 / 2<\alpha<1)$.

В случае оператора 1-го порядка на разлагаемую функцию $f(x)$ накладывались условия непрерывности, ограниченности вариации и выполнения двух простых функциональных соотношений

$$
f(x)=f(1-x), \quad f(x)=f(2 \alpha-x), \quad x \in[0, \alpha] .
$$

В случае оператора 2-го порядка на разлагаемую функцию, помимо условий гладкости на основном отрезке, накладывались условия аналитической продолжимости в некоторые треугольники и выполнения в этих треугольниках определенных функциональных соотношений.

В случае слабо нерегулярного дифференциального оператора 3-го порядка

$$
y^{\prime \prime \prime}-\lambda y, \quad a_{i} y^{(\nu)}(0)+y^{(\nu)}(1)=0, \quad \nu=0,1,2,
$$

где $a_{1}+a_{2}+a_{3}=0$ (здесь корни $\left\{\omega_{j}\right\}$ лежат в вершинах правильного треугольника), задача о разложении решена в [4]. При этом на разлагаемую функцию накладывались условия (и это по существу) аналитичности в некоторых многоугольниках комплексной плоскости и выполнения там функционального соотношения

$$
a f\left(\omega_{3} x\right)+b f\left(\omega_{1} x\right)+f(1-x)=0,
$$

где

$$
a=\left(a_{1}+a_{2} \varepsilon^{2}+a_{3} \varepsilon\right) / 3, \quad b=\left(a_{1}+a_{2} \varepsilon+a_{3} \varepsilon^{2}\right) / 3, \quad \varepsilon=\exp 2 \pi i / 3,
$$

при тех значениях $x \in[0,1]$, при которых аргументы функции $f$ попадают в упомянутые многоугольники.

Этот результат был распространен в [5] на случай оператора $n$-го порядка, где $n=4 k+1$, аналогичного оператору 3-го порядка. 
В статье автора [6] рассмотрен пучок 2-го порядка с простыми характеристиками

$$
\begin{gathered}
y^{\prime \prime}+p_{1} \lambda y^{\prime}+p_{2} \lambda^{2} y \\
\left(\alpha_{\nu 1} y^{\prime}(0)+\lambda \alpha_{\nu 2} y(0)\right)+\left(\beta_{\nu 1} y^{\prime}(1)+\lambda \beta_{\nu 2} y(1)\right)=0, \quad \nu=1,2,
\end{gathered}
$$

где $p_{j} \in \mathbb{C}$. Для корней $\omega_{1}, \omega_{2}$ этого пучка справедливо $0<\omega_{1}<\omega_{2}$. На коэффициенты $\alpha_{\nu j}, \beta_{\nu j} \in \mathbb{C}$ наложены условия, при которых рассматриваемый пучок является с.н. Получены достаточные условия сходимости двукратных разложений в.-ф. в биортогональные ряды по к.э. этого пучка (некоторые условия гладкости на разлагаемую в.-ф. и обращения в нуль в 0 и 1 компонент разлагаемой в.-ф. и их производных до некоторого порядка). Кроме того, найдено необходимое и достаточное условие на разлагаемую в.-ф. $f=\left(f_{1}, f_{2}\right)^{T}$, при котором имеет место представление в.-ф. этими рядами:

$$
f_{1}^{\prime}(x)-\omega_{2} f_{2}(x)=0 \quad \forall x \in[0,1] .
$$

Недавно в статье [7] изучались кратные разложения в.-ф. по к.э. регулярного пучка 4-го порядка с кратными характеристиками вида

$$
(d / d x-\lambda)^{4} y, \quad y^{(\nu)}(0)-y^{(\nu)}(1)=0, \quad \nu=0,1,2,3 .
$$

Получены достаточные условия 4-кратной разложимости в.-ф. по к.э. этого пучка (некоторые условия гладкости на разлагаемую в.-ф. и обращения в нуль в 0 и 1 компонент разлагаемой в.-ф. и их производных до некоторого порядка). Выполнение еще каких-то соотношений при этом не предполагалось.

В данной статье исследуются разложения в.-ф. по к.э. в случае с.н. пучка $L(\lambda)$ вида $(1)-(2)$ с кратными характеристиками.

\section{2. ВСПОМОГАТЕЛЬНЫЕ РЕЗУЛЬТАТЫ}

Лиинеаризуем задачу $L(\lambda)=0$ подстановкой $z_{1}=y, z_{2}=\lambda z_{1}$. С учетом (1)-(2) в результате получается краевая задача на с.з. уже для обыкновенного дифференциального оператора $\hat{L}$, но в пространстве в.-ф. $z=\left(z_{1}, z_{2}\right)^{T}$ :

$$
\hat{L} z-\lambda z=0
$$

где оператор $\hat{L}$ определяется дифференциальным выражением

$$
\hat{\ell}(z):=\left(\begin{array}{cc}
0 & 1 \\
-\frac{d^{2}}{d x^{2}} & 2 \frac{d}{d x}
\end{array}\right) z,
$$

действующем на множестве

$$
D_{\hat{L}}=\left\{\left(\begin{array}{c}
z_{1} \\
z_{2}
\end{array}\right) \mid z_{1}^{\prime}, z_{2} \in L_{1}[0,1], \hat{U}_{1}(z)=z_{1}(0)=0, \hat{U}_{2}(z)=z_{1}(1)-z_{1}^{\prime}(0)=0\right\}
$$

С.з. пучка $L(\lambda)$ и оператора $\hat{L}$, очевидно, совпадают, а система производных цепочек $L(\lambda)($ см. определение в [1, с. 102]) совпадает с системой к.э. оператора $\hat{L}$.

Обозначим через $\Gamma_{\nu}$ круговые контуры с центром в начале координат и радиуса $(2 \nu-1) \pi, \nu \in \mathbb{N}$. Хорошо известно, что

$$
-\frac{1}{2 \pi i} \int_{\Gamma_{\nu}} \hat{R}_{\lambda} f d \lambda
$$

где $\hat{R}_{\lambda}=(\hat{L}-\lambda E)^{-1}-$ резольвента оператора $\hat{L}$, есть частичная сумма разложений в.-ф. $f$ в биортогональный ряд Фурье по к.э. оператора $\hat{L}$, соответствующим тем с.з., которые попали внутрь контура $\Gamma_{\nu}$.

Пусть $\hat{R}_{\lambda} f=\left(z_{1}(x, \lambda ; f), z_{2}(x, \lambda ; f)\right)^{T}$ и $I_{s}(x, \nu ; f)=-\frac{1}{2 \pi i} \int_{\Gamma_{\nu}} z_{s}(x, \lambda ; f) d \lambda, s=1,2$.

Обозначим через $G(x, t, \lambda)$ функцию Грина пучка $L(\lambda)$. Для данной в.-ф. $f=\left(f_{1}, f_{2}\right)^{T}$ определим функцию $F(x, \lambda ; f):=-\lambda f_{1}(x)+2 f_{1}^{\prime}(x)-f_{2}(x)$.

Лемма 1. Если $f_{1}^{\prime}, f_{2} \in L_{1}[0,1]$, то при $\operatorname{Re} \lambda \leqslant 0$

$$
z_{1}(x, \lambda ; f)=\int_{0}^{1} G(x, t, \lambda) F(t, \lambda ; f) d t=\int_{0}^{x}(x-t) e^{\lambda(x-t)} F(t, \lambda ; f) d t+
$$




$$
+\frac{1}{\Delta_{0}^{-}(\lambda)} \int_{0}^{1} x(t-1) e^{\lambda(x+1-t)} F(t, \lambda ; f) d t=: z_{11}^{-}(x, \lambda ; f)+z_{12}^{-}(x, \lambda ; f),
$$

a npu $\operatorname{Re} \lambda \geqslant 0$

$$
\begin{gathered}
z_{1}(x, \lambda ; f)=\int_{0}^{1} G(x, t, \lambda) F(t, \lambda ; f) d t=\int_{x}^{1}(t-x) e^{\lambda(x-t)} F(t, \lambda ; f) d t+ \\
+\int_{0}^{1}(x-1) t e^{\lambda(x-t)} F(t, \lambda ; f) d t+\frac{1}{\Delta_{0}^{+}(\lambda)} \int_{0}^{1} x(t-1) e^{\lambda(x-1-t)} F(t, \lambda ; f) d t=: \\
=: z_{11}^{+}(x, \lambda ; f)+z_{12}^{+}(x, \lambda ; f)+z_{13}^{+}(x, \lambda ; f) \\
z_{2}(x, \lambda ; f)=\lambda z_{1}(x, \lambda ; f)+f_{1}(x) .
\end{gathered}
$$

Доказательство. Найдем резольвенту $\hat{R}_{\lambda}=(\hat{L}-\lambda E)^{-1}$. Для этого решим задачу $(\hat{L}-\lambda E) z=f$ относительно в.-ф. $z$ или, подробнее, задачу

$$
\left\{\begin{array}{l}
z_{2}-\lambda z_{1}=f_{1}, \\
-z_{1}^{\prime \prime}+2 z_{2}^{\prime}-\lambda z_{2}=f_{2},
\end{array} \quad \hat{U}_{j}(z)=0, \quad j=1,2 .\right.
$$

Выражая $z_{2}$ из первого уравнения системы в (7) и подставляя во второе (здесь требуется гладкость функции $f_{1}$ ) для нахождения $z_{1}$ получим краевую задачу для линейного неоднородного дифференциального уравнения 2-го порядка с постоянными коэффициентами:

$$
\ell\left(z_{1}, \lambda\right)=F(x, \lambda ; f), \quad \hat{U}_{j}(z)=0, \quad j=1,2 .
$$

Применяя метод вариации произвольных постоянных, находим общее решение дифференциального уравнения в (8) в случае $\operatorname{Re} \lambda \leqslant 0$ в виде

$$
z_{1}(x, \lambda)=C_{1} e^{\lambda x}+C_{2} x e^{\lambda x}+\int_{0}^{x}(x-t) e^{\lambda(x-t)} d t
$$

а в случае $\operatorname{Re} \lambda \geqslant 0-$ в виде

$$
z_{1}(x, \lambda)=C_{1} e^{\lambda x}+C_{2} x e^{\lambda x}-\int_{x}^{1}(x-t) e^{\lambda(x-t)} d t
$$

Затем находим константы $C_{1}, C_{2}$ из краевых условий (8) и в результате получим формулы (4)-(5). Формула (6) есть следствие первого соотношения в системе (7). Тем самым, лемма 1 доказана.

Пусть $K_{\delta}\left(\lambda_{k}\right)$ есть круги радиуса $\delta$ с центрами в $\lambda_{k}$ и $\mathbb{C}_{\delta}=\mathbb{C} \backslash\left(\cup_{k \in \mathbb{Z}} K_{\delta}\left(\lambda_{k}\right)\right)$. Обозначим через $\mathbb{C}_{\delta}^{+}\left(\mathbb{C}_{\delta}^{-}\right)$части $\mathbb{C}_{\delta}$, лежащие в правой (левой) полуплоскости. Используя те же рассуждения, что и в [1, с. 74-84], без труда устанавливаем справедливость леммы. В этой лемме и далее через $\Gamma_{\nu}^{-}$и $\Gamma_{\nu}^{+}$ обозначены части контура $\Gamma_{\nu}$, лежащие, соответственно, в левой и правой полуплоскости.

Лемма 2. Существует такая положительная константа $C_{\delta}$, ито

$$
\forall \lambda \in \mathbb{C}_{\delta}^{-}:\left|\Delta_{0}^{-}(\lambda)\right| \geqslant C_{\delta} ; \quad \forall \lambda \in \mathbb{C}_{\delta}^{+}:\left|\Delta_{0}^{+}(\lambda)\right| \geqslant C_{\delta} .
$$

Далее в доказательстве теоремы о разложении существенно используются оценки, даваемые следующей леммой (первоначальную сформулированную и доказанную в [6]).

Лемма 3. Если $\gamma(x, t)=\gamma_{0}+\gamma_{1} x+\gamma_{2} t\left(\gamma_{2} \neq 0\right) u \gamma(x, t) \operatorname{Re} \lambda \leqslant 0$ для всех $\lambda \in \Gamma_{\nu}^{+}\left(\Gamma_{\nu}^{-}\right)$nри $x \in[0,1]$, $t \in[a(x), b(x)]$, где $a(x), b(x)-$ заданные линейнье функции, $f \in L_{p}[0,1], p>1, \chi_{p}(\nu)=\nu^{1 / p}$ при $1<p<\infty u \chi_{\infty}(\nu)=\ln \nu$ nрu $p=\infty$, mo

$$
\left|-\frac{1}{2 \pi i} \int_{\Gamma_{\nu}+\left(\Gamma_{\nu}^{-}\right)}\left(\int_{a(x)}^{b(x)} e^{\gamma(x, t) \lambda} h(t) d t\right) d \lambda\right| \leqslant C\|h\|_{p} \chi_{p}(\nu),
$$

где обозначено $\|\cdot\|_{p}=\|\cdot\|_{L_{p}[0,1]}$.

Отметим, что аналогичные оценки впервые использовались в [8]. 


\section{3. ФОРМУЛИРОВКА И ДОКАЗАТЕЛЬСТВО ТЕОРЕМ О РАЗЛОЖЕНИИ}

Справедлива следующая теорема о разложении.

Теорема 1. Если в.-ф. $f=\left(f_{1}, f_{2}\right)^{T}$ удовлетворяет условиям:

$$
\begin{gathered}
f_{1}^{\prime \prime \prime}, f_{2}^{\prime \prime} \in L_{p}[0,1], \quad 1<p \leqslant+\infty \\
f_{1}^{(s)}(0)=f_{1}^{(s)}(1)=0, \quad s=0,1,2 ; \quad f_{2}^{(s)}(0)=f_{2}^{(s)}(1)=0, \quad s=0,1 .
\end{gathered}
$$

то справедливы формулы двукратного разложения в.-ф. $f$ по к.э. пучка $L(\lambda)$ :

$$
\lim _{\nu \rightarrow+\infty} I_{s}(x, \nu ; f)=f_{s}(x)+(1-x)\left(x f_{1}^{\prime}(x)-f_{1}(x)-x f_{2}(x)\right)^{(s-1)}, \quad s=1,2,
$$

где сходимость равномерная по $x \in[0,1]$.

Доказательство. Рассмотрим $I_{1}(x, \nu ; f)$. Справедливо представление

$$
I_{1}(x, \nu ; f)=-\frac{1}{2 \pi i} \int_{\Gamma_{\nu}^{-}} z_{1}(x, \lambda ; f) d \lambda-\frac{1}{2 \pi i} \int_{\Gamma_{\nu}^{+}} z_{1}(x, \lambda ; f) d \lambda=: I_{1}^{-}(x, \nu ; f)+I_{1}^{+}(x, \nu ; f) .
$$

Найдем асимптотику $I_{1}^{-}(x, \nu ; f)$ при $\nu \rightarrow+\infty$. В соответствии с формулой (4)

$$
I_{1}^{-}(x, \nu ; f)=-\frac{1}{2 \pi i} \int_{\Gamma_{\nu}^{-}} z_{11}^{-}(x, \lambda ; f) d \lambda-\frac{1}{2 \pi i} \int_{\Gamma_{\nu}^{-}} z_{12}^{-}(x, \lambda ; f) d \lambda=: I_{11}^{-}(x, \nu ; f)+I_{12}^{-}(x, \nu ; f) .
$$

Для выражения при экспоненте под знаком интеграла в $z_{11}^{-}(x, \lambda ; f)$ имеем:

$$
(x-t) F(t, \lambda ; f)=\lambda\left((t-x) f_{1}(t)\right)+\left((x-t)\left(2 f_{1}^{\prime}(t)-f_{2}(t)\right)=: \lambda a_{111}(x, t)+a_{112}(x, t) .\right.
$$

Следовательно,

$$
z_{11}^{-}(x, \lambda ; f)=\lambda \int_{0}^{x} e^{\lambda(x-t)} a_{111}(x, t) d t+\int_{0}^{x} e^{\lambda(x-t)} a_{112}(x, t) d t=: \lambda A_{111}(x, \lambda ; f)+A_{112}(x, \lambda ; f) .
$$

Справедливы формулы

$$
\left.\begin{array}{c}
a_{112, t}^{\prime}(x, t)=-\left(2 f_{1}^{\prime}(t)-f_{2}(t)\right)+(x-t)\left(2 f_{1}^{\prime \prime}(t)-f_{2}^{\prime}(t)\right), \\
a_{112, t^{2}}^{\prime \prime}(x, t)=-\left(4 f_{1}^{\prime \prime}(t)-2 f_{2}^{\prime}(t)\right)+(x-t)\left(2 f_{1}^{\prime \prime \prime}(t)-f_{2}^{\prime \prime}(t)\right),
\end{array}\right\}
$$

Проинтегрируем по частям $A_{112}(x, \lambda ; f)$ один раз, а $A_{111}(x, \lambda ; f)$ два раза. При этом воспользуемся формулами (17), (18) и предположениями (11), (12). Получим:

$$
\begin{gathered}
A_{112}(x, \lambda ; f)=\frac{1}{\lambda} \int_{0}^{x} e^{\lambda(x-t)} a_{112, t}^{\prime}(x, t) d t \\
\lambda A_{111}(x, \lambda ; f)=-\frac{1}{\lambda} f_{1}(x)+\frac{1}{\lambda} \int_{0}^{x} e^{\lambda(x-t)} a_{111, t^{2}}^{\prime \prime}(x, t) d t .
\end{gathered}
$$

Так как подынтегральные функции ограничены при $0 \leqslant t \leqslant x, x \in[0,1]$, то по лемме 3

$$
\left|-\frac{1}{\lambda} \int_{\Gamma_{\nu}^{-}} A_{112}(x, \lambda ; f) d \lambda\right| \leqslant C\left(\left\|f_{2}^{\prime}\right\|_{\infty}+\left\|f_{1}^{\prime \prime}\right\|_{\infty}\right) \frac{\chi_{\infty}(\nu)}{\nu} \rightarrow 0, \quad \nu \rightarrow+\infty,
$$

расномерно по $x \in[0,1]$. Следовательно,

$$
A_{112}(x, \lambda ; f)=\alpha(x, \lambda ; f)
$$


где через $\alpha(x, \lambda ; f)$ здесь и далее будет обозначаться любая функция, для которой

$$
\int_{\Gamma_{\nu}^{ \pm}} \alpha(x, \lambda ; f) d \lambda=o(1), \quad \nu \rightarrow+\infty
$$

равномерно по $x \in[0,1]$. Далее, не оговаривая это особо, считаем $o(1)$ равномерной по $x \in[0,1]$.

Для $A_{111}(x, \lambda ; f)$ аналогично получим:

$$
\lambda A_{111}(x, \lambda ; f)=-\frac{1}{\lambda} f_{1}(x)+\alpha(x, \lambda ; f) .
$$

Таким образом, из (15), (16), (21), (22) следует асимптотика

$$
I_{11}^{-}(x, \nu ; f)=\frac{1}{2} f_{1}(x)+o(1), \quad \nu \rightarrow+\infty .
$$

Рассмотрим теперь $I_{12}^{-}(x, \nu ; f)$. Для выражения при экспоненте под знаком интеграла в $z_{12}^{-}(x, \lambda ; f)$ имеем:

$$
x(t-1) F(t, \lambda ; f)=\lambda\left(x(1-t) f_{1}(t)\right)+\left(x(t-1)\left(2 f_{1}^{\prime}(t)-f_{2}(t)\right)=: \lambda a_{121}(x, t)+a_{122}(x, t) .\right.
$$

Следовательно,

$$
\begin{gathered}
z_{12}^{-}(x, \lambda ; f)=\frac{\lambda}{\Delta_{0}^{-}(\lambda)} \int_{0}^{1} e^{\lambda(x+1-t)} a_{121}(x, t) d t+\frac{1}{\Delta_{0}^{-}(\lambda)} \int_{0}^{1} e^{\lambda(x+1-t)} a_{122}(x, t) d t=: \\
=: \lambda A_{121}(x, \lambda ; f)+A_{122}(x, \lambda ; f)
\end{gathered}
$$

Справедливы формулы

$$
\left.\begin{array}{c}
a_{122, t}^{\prime}(x, t)=x\left(2 f_{1}^{\prime}(t)-f_{2}(t)\right)+x(t-1)\left(2 f_{1}^{\prime \prime}(t)-f_{2}^{\prime}(t)\right), \\
a_{122, t^{2}}^{\prime \prime}(x, t)=x\left(4 f_{1}^{\prime \prime}(t)-2 f_{2}^{\prime}(t)\right)+x(t-1)\left(2 f_{1}^{\prime \prime \prime}(t)-f_{2}^{\prime \prime}(t)\right),
\end{array}\right\}
$$

Проинтегрируем по частям $A_{122}(x, \lambda ; f)$ один раз, а $A_{121}(x, \lambda ; f)$ два раза. При этом воспользуемся формулами (25), (26), предположениями (11), (12) и леммой 2. Получим:

$$
\begin{gathered}
A_{122}(x, \lambda ; f)=\frac{1}{\lambda \Delta_{0}^{-}(\lambda)} \int_{0}^{1} e^{\lambda(x+1-t)} a_{122, t}^{\prime}(x, t) d t=\alpha(x, \lambda ; f), \\
\lambda A_{121}(x, \lambda ; f)=-\frac{1}{\lambda \Delta_{0}^{-}} \int_{0}^{1} e^{\lambda(x+1-t)} a_{121, t^{2}}^{\prime \prime}(x, t) d t=\alpha(x, \lambda ; f) .
\end{gathered}
$$

Таким образом, из (15), (24), (9), (27), (28) следует асимптотика

$$
I_{12}^{-}(x, \nu ; f)=o(1), \quad \nu \rightarrow+\infty .
$$

Следовательно, на основании (15), (23), (29)

$$
I_{1}^{-}(x, \nu ; f)=\frac{1}{2} f_{1}(x)+o(1), \quad \nu \rightarrow+\infty .
$$

Найдем теперь асимптотику $I_{1}^{+}(x, \nu ; f)$ при $\nu \rightarrow+\infty$. В соответствии с формулой (5)

$$
\begin{gathered}
I_{1}^{+}(x, \nu ; f)=-\frac{1}{2 \pi i} \int_{\Gamma_{\nu}^{+}} z_{11}^{+}(x, \lambda ; f) d \lambda-\frac{1}{2 \pi i} \int_{\Gamma_{\nu}^{+}} z_{12}^{+}(x, \lambda ; f) d \lambda- \\
-\frac{1}{2 \pi i} \int_{\Gamma_{\nu}^{+}} z_{13}^{+}(x, \lambda ; f) d \lambda=: I_{11}^{+}(x, \nu ; f)+I_{12}^{+}(x, \nu ; f)+I_{13}^{+}(x, \nu ; f) .
\end{gathered}
$$


Аналогично предыдущему (см. (23), (29)) получим:

$$
\begin{gathered}
I_{11}^{+}(x, \nu ; f)=\frac{1}{2} f_{1}(x)+o(1), \quad \nu \rightarrow+\infty, \\
I_{13}^{+}(x, \nu ; f)=o(1), \quad \nu \rightarrow+\infty .
\end{gathered}
$$

Осталось рассмотреть интеграл $I_{12}^{+}(x, \nu ; f)$.

Для выражения при экспоненте под знаком интеграла в $z_{12}^{+}(x, \lambda ; f)$ имеем:

$$
(x-1) t F(t, \lambda ; f)=\lambda\left((1-x) t f_{1}(t)\right)+\left((x-1) t\left(2 f_{1}^{\prime}(t)-f_{2}(t)\right)=: \lambda b_{121}(x, t)+b_{122}(x, t) .\right.
$$

Следовательно,

$$
z_{12}^{+}(x, \lambda ; f)=\lambda \int_{0}^{1} e^{\lambda(x-t)} b_{121}(x, t) d t+\int_{0}^{1} e^{\lambda(x-t)} b_{122}(x, t) d t=: \lambda B_{121}(x, \lambda ; f)+B_{122}(x, \lambda ; f) .
$$

Справедливы формиулы

$$
\left.\begin{array}{c}
b_{122, t}^{\prime}(x, t)=(x-1)\left(2 f_{1}^{\prime}(t)-f_{2}(t)+2 t f_{1}^{\prime \prime}(t)-t f_{2}^{\prime}(t)\right), \\
b_{122, t^{2}}^{\prime \prime}(x, t)=(x-1)\left(4 f_{1}^{\prime \prime}(t)-2 f_{2}^{\prime}(t)+2 t f_{1}^{\prime \prime \prime}(t)-t f_{2}^{\prime \prime}(t)\right),
\end{array}\right\}
$$

Проинтегрируем по частям $B_{122}(x, \lambda ; f)$ один раз, а $B_{121}(x, \lambda ; f)$ два раза. При этом воспользуемся формулами (35), (36) и предположениями (11), (12). Получим:

$$
\begin{aligned}
B_{112}(x, \lambda ; f) & =\frac{1}{\lambda} \int_{0}^{1} e^{\lambda(x-t)} b_{122, t}^{\prime}(x, t) d t=\frac{1}{\lambda} \int_{0}^{x} e^{\lambda(x-t)} b_{122, t}^{\prime}(x, t) d t+\alpha(x, \lambda ; f), \\
\lambda B_{121}(x, \lambda ; f) & =\frac{1}{\lambda} \int_{0}^{1} e^{\lambda(x-t)} b_{121, t^{2}}^{\prime \prime}(x, t) d t=\frac{1}{\lambda} \int_{0}^{x} e^{\lambda(x-t)} b_{121, t^{2}}^{\prime \prime}(x, t) d t+\alpha(x, \lambda ; f) .
\end{aligned}
$$

Из (34), (37), (38) будем иметь:

$$
z_{12}^{+}(x, \lambda ; f)=\frac{1}{\lambda} \int_{0}^{x} e^{\lambda(x-t)}\left(b_{122, t}^{\prime}(x, t)+b_{121, t^{2}}^{\prime \prime}(x, t)\right) d t+\alpha(x, \lambda ; f) .
$$

Используя аддитивность интеграла, теорию вычетов и предположения (12), получим:

$$
\begin{gathered}
I_{12}^{+}(x, \nu ; f)=-\frac{1}{2 \pi i} \int_{\Gamma_{\nu}} \frac{1}{\lambda} \int_{0}^{x} e^{\lambda(x-t)}\left(b_{122, t}^{\prime}(x, t)+b_{121, t^{2}}^{\prime \prime}(x, t)\right) d t+ \\
+\frac{1}{2 \pi i} \int_{\Gamma_{\nu}^{-}} \frac{1}{\lambda} \int_{0}^{x} e^{\lambda(x-t)}\left(b_{122, t}^{\prime}(x, t)+b_{121, t^{2}}^{\prime \prime}(x, t)\right) d t+o(1)= \\
=-\int_{0}^{x}\left(b_{122, t}^{\prime}(x, t)+b_{121, t^{2}}^{\prime \prime}(x, t)\right) d t+o(1)=-\left(b_{122, t}^{\prime}(x, x)+b_{121, t^{2}}^{\prime \prime}(x, x)\right)+o(1)= \\
=(1-x)\left(x f_{1}^{\prime}(x)-f_{1}(x)-x f_{2}(x)\right)+o(1), \quad \nu \rightarrow+\infty .
\end{gathered}
$$

Следовательно, на основании (31)-(33), (39)

$$
I_{1}^{+}(x, \nu ; f)=\frac{1}{2} f_{1}(x)+(1-x)\left(x f_{1}^{\prime}(x)-f_{1}(x)-x f_{2}(x)\right)+o(1), \quad \nu \rightarrow+\infty .
$$

С учетом (14), (30) и (40)

$$
I_{1}(x, \nu ; f)=\frac{1}{2} f_{1}(x)+(1-x)\left(x f_{1}^{\prime}(x)-f_{1}(x)-x f_{2}(x)\right)+o(1), \quad \nu \rightarrow+\infty
$$

что доказывает (13) для $s=1$. 
Рассмотрим теперь $I_{2}(x, \nu ; f)$. Справедливо представление

$$
\begin{gathered}
\left.I_{2}(x, \nu ; f)\right)=-\frac{1}{2 \pi i} \int_{\Gamma_{\nu}} \lambda z_{1}(x, \lambda ; f) d \lambda-\frac{1}{2 \pi i} \int_{\Gamma_{\nu}} f_{1}(x) d \lambda=-\frac{1}{2 \pi i} \int_{\Gamma_{\nu}} \lambda z_{1}(x, \lambda ; f) d \lambda= \\
=-\frac{1}{2 \pi i} \int_{\Gamma_{\nu}^{-}} \lambda z_{1}(x, \lambda ; f) d \lambda-\frac{1}{2 \pi i} \int_{\Gamma_{\nu}^{+}} \lambda z_{1}(x, \lambda ; f) d \lambda=: I_{2}^{-}(x, \nu ; f)+I_{2}^{+}(x, \nu ; f) .
\end{gathered}
$$

Отсюда видно, как изменятся рассуждения при исследовании $I_{2}(x, \nu ; f)$ по сравнению с исследованием $I_{1}(x, \nu ; f)$. Эти изменения вызваны присутствием лишнего множителя $\lambda$ у каждого слагаемого в (41).

Найдем асимптотику $I_{2}^{-}(x, \nu ; f)$ при $\nu \rightarrow+\infty$. В соответствии с $(41)$ и (15) имеем:

$$
I_{2}^{-}(x, \nu ; f)=-\frac{1}{2 \pi i} \int_{\Gamma_{\nu}^{-}} \lambda z_{11}^{-}(x, \lambda ; f) d \lambda-\frac{1}{2 \pi i} \int_{\Gamma_{\nu}^{-}} \lambda z_{12}^{-}(x, \lambda ; f) d \lambda=: I_{21}^{-}(x, \nu ; f)+I_{22}^{-}(x, \nu ; f) .
$$

На основании (16)

$$
\lambda z_{11}^{-}(x, \lambda ; f)=\lambda^{2} A_{111}(x, \lambda ; f)+\lambda A_{112}(x, \lambda ; f) .
$$

Чтобы погасить дополнительный множитель $\lambda$, проинтегрируем по частям $A_{112}(x, \lambda ; f)$ не один раз, как раньше, а два раза, а $A_{111}(x, \lambda ; f)$ три раза. Воспользовавшись формулами (17), (18), предположениями (11), (12), леммой 3 (формула (10) применяется для р из (11)) и формулами (19), (20), получим:

$$
\begin{aligned}
\lambda A_{112}(x, \lambda ; f)= & -\frac{1}{\lambda} f_{2}(x)+\frac{2}{\lambda} f_{1}^{\prime}(x)+\frac{1}{\lambda} \int_{0}^{x} e^{\lambda(x-t)} a_{112, t^{2}}^{\prime \prime}(x, t) d t= \\
& =-\frac{1}{\lambda} f_{2}(x)+\frac{2}{\lambda} f_{1}^{\prime}(x)+\alpha(x, \lambda ; f), \\
\lambda^{2} A_{111}(x, \lambda ; f)= & -f_{1}(x)-\frac{2}{\lambda} f_{1}^{\prime}(x)+\frac{1}{\lambda} \int_{0}^{x} e^{\lambda(x-t)} a_{111, t^{3}}^{\prime \prime \prime}(x, t) d t= \\
& =-f_{1}(x)-\frac{2}{\lambda} f_{1}^{\prime}(x)+\alpha(x, \lambda ; f) .
\end{aligned}
$$

Следовательно, на основании (42)-(45) имеем:

$$
I_{21}^{-}(x, \nu ; f)=\frac{1}{2} f_{2}(x)+\frac{1}{2 \pi i} \int_{\Gamma_{\nu}^{-}} f_{1}(x) d \lambda+o(1), \quad \nu \rightarrow+\infty .
$$

Аналогично предыдущему

$$
I_{22}^{-}(x, \nu ; f)=o(1), \quad \nu \rightarrow+\infty .
$$

С учетом (42) из (46), (47) получим:

$$
I_{2}^{-}(x, \nu ; f)=\frac{1}{2} f_{2}(x)+\frac{1}{2 \pi i} \int_{\Gamma_{\nu}^{-}} f_{1}(x) d \lambda+o(1), \quad \nu \rightarrow+\infty .
$$

Найдем теперь асимптотику $I_{2}^{+}(x, \nu ; f)$ при $\nu \rightarrow+\infty$. В соответствии с формулами $(41),(5),(4)$ имеем:

$$
\begin{aligned}
& I_{2}^{+}(x, \nu ; f)=-\frac{1}{2 \pi i} \int_{\Gamma_{\nu}^{+}} \lambda z_{11}^{+}(x, \lambda ; f) d \lambda-\frac{1}{2 \pi i} \int_{\Gamma_{\nu}^{+}} \lambda z_{12}^{+}(x, \lambda ; f) d \lambda- \\
& -\frac{1}{2 \pi i} \int_{\Gamma_{\nu}^{+}} \lambda z_{13}^{+}(x, \lambda ; f) d \lambda=: I_{21}^{+}(x, \nu ; f)+I_{22}^{+}(x, \nu ; f)+I_{23}^{+}(x, \nu ; f) .
\end{aligned}
$$

Аналогично предыдущему в силу предположений (11), (12), лемм 2 и 3, свойств интегралов в комплексной плоскости, теории вычетов и формул (35), (36) получим:

$$
\begin{gathered}
I_{21}^{+}(x, \nu ; f)=\frac{1}{2} f_{2}(x)+\frac{1}{2 \pi i} \int_{\Gamma_{\nu}^{+}} f_{1}(x) d \lambda+o(1), \quad \nu \rightarrow+\infty \\
I_{22}^{+}(x, \nu ; f)=-b_{122, t}^{\prime}(x, x)-b_{121, t^{2}}^{\prime \prime}(x, x)+o(1)= \\
=(1-x)\left(x f_{1}^{\prime}(x)-f_{1}(x)-x f_{2}^{\prime}(x)\right)^{\prime}+o(1), \quad \nu \rightarrow+\infty
\end{gathered}
$$




$$
I_{23}^{+}(x, \nu ; f)=o(1), \quad \nu \rightarrow+\infty
$$

Из (49)-(52) получим:

$$
I_{2}^{+}(x, \nu ; f)=\frac{1}{2} f_{2}(x)+\frac{1}{2 \pi i} \int_{\Gamma_{\nu}^{+}} f_{1}(x) d \lambda+(1-x)\left(x f_{1}^{\prime}(x)-f_{1}(x)-x f_{2}^{\prime}(x)\right)^{\prime}+o(1), \quad \nu \rightarrow+\infty .
$$

Таким образом, на основании (41), (48), (53) справедливо равенство

$$
\begin{gathered}
I_{2}(x, \nu ; f)=f_{2}(x)+\frac{1}{2 \pi i} \int_{\Gamma_{\nu}} f_{1}(x) d \lambda+(1-x)\left(x f_{1}^{\prime}(x)-f_{1}(x)-x f_{2}^{\prime}(x)\right)^{\prime}+o(1)= \\
=f_{2}(x)+(1-x)\left(x f_{1}^{\prime}(x)-f_{1}(x)-x f_{2}^{\prime}(x)\right)^{\prime}+o(1), \quad \nu \rightarrow+\infty,
\end{gathered}
$$

а это и есть утверждение (13) в случае $s=2$.

Из этой теоремы, как простое следствие, получаем необходимое и достаточное условие разложения в.-ф. $f=\left(f_{1}, f_{2}\right)^{T}$ в биортогональный ряд фурье по к.э. пучка $L(\lambda)$.

Теорема 2. Пусть в.-ф. $f$ удовлетворяет условиям (11), (12). Для того итобы имели место формуль двукратного разложения в.-ф. $f$ по корневым элементам пучка $L(\lambda)$ с равномерной сходимостью по $x \in[0,1]$

$$
\lim _{\nu \rightarrow+\infty} I_{s}(x, \nu ; f)=f_{s}(x), \quad s=1,2,
$$

необходимо и достаточно, чтобы выполнялось следующее тождество на $[0,1]$

$$
x f_{1}^{\prime}(x)-f_{1}(x)-x f_{2}(x) \equiv 0 .
$$

Работа подготовлена в рамках выполнения государственного задания Минобрнауки России (проект № 1.1520.2014/K).

\section{Библиографический список}

1. Наймарк М. А. Линейные дифференциальные операторы. М. : Наука, 1969. 528 с.

2. Шкаликов А. А. Краевые задачи для обыкновенных дифференциальных уравнений с параметром в граничных условиях // Тр. семинара им. И. Г. Петровского. М. : Изд-во Моск. ун-та, 1983. № 9. С. 190-229.

3. Хромов А. П., Гуревич А. П. Операторы дифференцирования первого и второго порядков со знакопеременной весовой функцией // Матем. заметки. 1994. Т. 56, вып. 1. С. 3-15.

4. Хромов А. П. Разложение по собственным функциям одной краевой задачи третьего порядка // Исследования по теории операторов. Уфа, 1988. C. $182-193$.

5. Дмитриев О. Ю. Разложение по собственным функциям дифференциального оператора $n$-го порядка с нерегулярными краевыми условиями //
Изв. Сарат. ун-та. Нов. сер. Сер. Математика. Механика. Информатика. 2007. Т. 7, вып. 2. С. 10-14.

6. Рыхлов В. С. Разложение по собственным функциям квадратичных сильно нерегулярных пучков дифференциальных операторов второго порядка // Изв. Сарат. ун-та. Нов. сер. Сер. Математика. Механика. Информатика. 2013. Т. 13, вып. 1, ч. 1. C. 21-26.

7. Вагабов А. И., Абуд А. Х. Четырехкратная разложимость в ряды Фурье по корневым элементам дифференциального пучка с четырехкратной характеристикой // Вестн. Дагест. гос. ун-та. 2015. Т. 30, вып. 1. С. 34-39.

8. Хромов А. П. Теоремы равносходимости для интегро-дифференциальных и интегральных операторов // Матем. сб. 1981. Т. 114(156), № 3. C. $378-405$.

\section{Expansion in Root Functions of Strongly Irregular Pencil of Differential Operators of the Second Order with Multiple Characteristics}

\section{S. Rykhlov}

Victor S. Rykhlov, Saratov State University, 83, Astrakhanskaya st., 410012, Saratov, Russia, RykhlovVS@yandex.ru

We consider the quadratic strongly irregular pencil of ordinary second order differential operators with constant coefficients and with a multiple root of the characteristic equation. The amounts of double expansions in biorthogonal Fourier series in the derived chains of such pencils and a necessary and sufficient condition for convergence of these expansions to the expanded vector-valued function are found. This necessary and sufficient condition is a differential equation relating the components of the expanded vector function. At the same time some conditions of smoothness on the components of the expanded vector-valued function and requirements of the vanishing of its components and some of their derivatives at the ends of the main segment are imposed. 
Key words: quadratic pencil of differential operators, multiple characteristic, multiple root of characteristic equation, strongly irregular pencil, two-fold expansion in the eigenfunctions, two-fold expansion in the root elements, biorthogonal series on root elements, derived chains, conditions of multiple expandability.

The results were obtained within the framework of the state task of Russian Ministry of Education and Science (project no. 1.1520.2014K).

\title{
References
}

1. Naimark M. A. Linear Differential Operators. Pt. I. New York, F. Ungar Publ. Co., 1967, 144 p.; Pt. II. New York, F. Ungar Publ. Co., 1968, 352 p. (Russ. ed.: Naimark M. A. Linear Differential Operators. Moscow, Nauka, 1968, 528 p.).

2. Shkalikov A. A. Boundary value problems for ordinary differential equations with a parameter in the boundary conditions. J. Soviet Math., 1986, vol. 33, iss. 6 , pp. 1311-1342.

3. Gurevich A. P., Khromov A. P. First and second order differential operators with weight functions of variable sign. Math. Notes, 1994, vol. 56, iss. 1, pp. 653-661.

4. Khromov A. P. Razlozhenie po sobstvennym funktsiiam odnoi kraevoi zadachi tret'ego poriadka [Expansion in the eigenfunctions of a boundary value problem of the third order]. Issledovaniia po teorii operatorov [Researches on the theory of operators], Ufa, 1988, pp. 182-193 (in Russian).

5. Dmitriev O. Iu. Expansion on eigenfunctions the differential operator of $n$-th order with irregular boundary conditions. Izv. Saratov Univ. (N. S.), Ser. Math. Mech. Inform., 2007, vol. 7, iss. 2, pp. 10-14 (in Russian).

6. Rykhlov V. S. Expansion in eigenfunctions of quadratic strongly irregular pencils of differential operators of the second order. Izv. Saratov Univ. (N. S.), Ser. Math. Mech. Inform., 2013, vol. 13, iss. 1, part 1, pp. 21-26 (in Russian).

7. Vagabov A. I., Abud A. Kh. Quadruple expandability in Fourier's series on root elements of a differential pencil with quadruple characteristic. Vestnik Dagest. gos. un-ta [Bull. of the Dagestan State Univ.], 2015, vol. 30, iss. 1, pp. 34-39.

8. Hromov A. P. Equiconvergence theorems for integrodifferential and integral operators. Math. USSR Sb., 1982, vol. 42 , iss. 3, pp. 331-355.

УДК 517.54

\section{ОДНОРОДНАЯ КРАЕВАЯ ЗАДАЧА ГИЛЬБЕРТА С БЕСКОНЕЧНЫМ ИНДЕКСОМ НА ОКРУЖНОСТИ}

\begin{abstract}
А. Х. Фатыхов ${ }^{1}$, П. Л. Шабалин ${ }^{2}$
${ }^{1}$ Фатыхов Азат Халитович, аспирант кафредры высшей математики, Казанский государственный архитектурно-строительный университет, vitofat@gmail.com

${ }^{2}$ Шабалин Павел Леонидович, доктор физико-математических наук, просрессор кафредры высшей математики, Казанский государственный архитектурно-строительный университет, pavel.shabalin@ mail.ru

В статье рассматривается краевая задача Гильберта теории аналитических фрункцй с бесконечным индексом и краевым условием на окружности, коэсрфициенты краевого условия непрерывны по Гельдеру всюду, кроме одной особой точки, в которой аргумент фрункции коэсрфициентов имеет разрыв второго рода (степенного порядка с показателем, меньше единицы). В такой постановке задача с бесконечным индексом рассматривается впервые. Получены срормулы общего решения однородной задачи, исследованы вопросы существования и единственности решения, описано множество решений в случае неединственности. При исследовании решения применялся аппарат теории целых функций и геометрической теории фрункций комплексного переменного.
\end{abstract}

Ключевые слова: задача Гильберта, бесконечный индекс, целые фрункции, индикатор роста.

DOI: $10.18500 / 1816-9791-2016-16-2-174-180$

\section{1. ПОСТАНОВКА ЗАДАЧИ}

Пусть $D=\left\{z=r e^{i \theta}: 0<r<1,0 \leqslant \theta<2 \pi\right\}-$ единичный круг в плоскости комплексного переменного $z, L=\partial D$. Рассмотрим краевую задачу Гильберта теории аналитических функций с краевым условием на единичной окружности

$$
a(t) \operatorname{Re} \Phi(t)-b(t) \operatorname{Im} \Phi(t)=c(t), \quad t \in L, \quad t \neq 1,
$$

